\title{
Matching Decision between Organizational Innovation and Technological Innovation: Based on M Enterprise
}

\section{Wang Chenggang*}

School of Economics and Business Administration, Heilongjiang University, Xuefu Road, Harbin, China

Keywords: Organizational Innovation; Technological Innovation; Matching Decision; System Simulation

\begin{abstract}
With the increasing of managers emphasis on enterprise innovation, the problem of matching decision relationship between organizational innovation (OI) and technological innovation (TI) gets more and more attention gradually. In this background, this paper chooses the method of case analysis, matching evaluation matrix, system dynamics simulation, system analysis. The research introduces the development environment, the OI and TI status of $\mathrm{M}$ enterprise. Furthermore, this paper researches the matching decision status between OI and TI in the view of static and dynamic, and also describes the matching decision route. At last, this paper proposes some advices to promote the manage activities on the matching decision between OI and TI of M enterprise, and also provides important reference.
\end{abstract}

\section{Introduction}

In today's enterprise innovation management, more and more researchers have begun to pay attention to the problem of matching organizational innovation with technological innovation ${ }^{[1]}$. And from the practice of enterprise development, it can be seen that the decision-making status of corporate organizational innovation and technological innovation also has a relatively important impact on the realization of the overall performance of the enterprise ${ }^{[2]}$. Therefore, this article takes the high-end equipment manufacturing company $\mathrm{M}$ enterprise as a case to study the matching decision between $M$ enterprise's organizational innovation and technological innovation. On the one hand, it enriches the theory of enterprise management research and reflects the theoretical significance of this research ${ }^{[3]}$. On the other hand, the research provides an important reference basis for $\mathrm{M}$ firm during the management process and reflects its practical significance.

\section{Basic profile of $M$ company}

Company $\mathrm{M}$ was founded in accordance with the overall deployment of China's national defense industry and is also a state-owned ultra-large high-tech equipment manufacturing company officially approved by the State Council. In terms of the company's specific business development, $M$ firm's main business projects include research and development of launch vehicles, space manned spacecraft, satellites, space station construction, deep space aircraft research and development of space products, military missiles and other military products, as well as information technology products, new materials or related products such as new energy. The related products involved in company $\mathrm{M}$ are all high-end equipment products. Company $\mathbf{M}$ is a typical high-end equipment manufacturing enterprise.

Based on the long-term development history of the current $\mathrm{M}$ enterprise, they have further accumulated experience in organizational development and technology research and development to varying degrees. It has played a huge role in promoting enterprise $\mathrm{OI}$ and $\mathrm{TI}{ }^{[4]}$. But the more complex the organizational structure, the more difficult it is to manage; the more high-level technology companies, the more difficult it is to achieve a long-term match between TI and $\mathrm{OI}^{[5]}$. And with the continuous and rapid changes and comprehensive improvement of the respective levels and capabilities of TI and OI of M enterprises, it has also led to a large degree of mismatch between OI and TI of M enterprises. Therefore, in the state where the match between OI and TI of 
M enterprises is relatively poor, it will naturally hinder the improvement of the overall innovation ability of enterprises. However, under this situation, the relevant managers of enterprise $\mathrm{M}$ still have problems such as inadequate experience and improper measures for judging the matching status between OI and TI and optimizing the matching status between OI and TI. Therefore, this research focuses on the matching decision between OI and TI of $\mathrm{M}$ companies, and conducts a comprehensive and thorough research, and proposes more targeted improvement measures and suggestions in all aspects, so as to improve the decision management of OI and TI matching of M companies Relevant managers provide important reference when making management decisions, and finally provide important support for comprehensively promoting the improvement of $\mathrm{M}$ enterprise's innovation ability, laying a solid foundation for development.

\section{OI and TI Static Matching Decision in M Enterprise}

The relevant empirical analysis steps based on the matching evaluation matrix ${ }^{[6]}$ bring the relevant research data on the MI OI and TI and their matching decision-making in this case into the satisfaction formula, and adjust and transform the relevant data. Thus, a single-objective optimization model for the matching decision between OI and TI of M enterprise is obtained:

$$
\max F=0.5 \sum_{i=1}^{4} \sum_{j=1}^{2} \theta_{i j} y_{i j}+0.5 \sum_{i=1}^{4} \sum_{j=1}^{2} \psi_{i j} y_{i j}=\sum_{i=1}^{4} \sum_{j=1}^{2} f_{i j} y_{i j}
$$

$$
\begin{gathered}
\text { s.t. } \sum_{\mathrm{j}=1}^{2} y_{i j}=1, \quad i=1,2, \ldots, 4 ;(1) \\
\sum_{\mathrm{i}=1}^{4} y_{i j} \leq 1, \quad, \quad j=1,2 ;(2) \\
y_{i j}=0 \text { or } 1, \quad i=1,2, \ldots, 4, j=1,2 .(3)
\end{gathered}
$$

Among them $f_{i j}=0.5 \theta_{i j}+0.5 \psi_{i j}$, coefficient matrix $\left[f_{i j}\right]_{4 \times 2}$, As shown in Table 1 。

Tab 1 Coefficient matrix $\left[f_{i j}\right]_{4 \times 2}$

\begin{tabular}{|c|c|c|}
\hline$f_{i j}$ & $B_{1}$ & $B_{2}$ \\
\hline$A_{1}$ & 0.841 & 0.702 \\
\hline$A_{2}$ & 0.957 & $-K^{\prime}$ \\
\hline$A_{3}$ & $-K^{\prime}$ & $-K^{\prime}$ \\
\hline$A_{4}$ & 0.7125 & $-K^{\prime}$ \\
\hline
\end{tabular}

The following single-objective optimization model can be solved by programming the Lingo9.0 optimization software package:

$$
Y_{M}^{*}=\left[y_{i j}^{*}\right]_{4 \times 2}=\left[\begin{array}{ll}
1 & 1 \\
1 & 0 \\
1 & 0 \\
0 & 1
\end{array}\right](4)
$$

It can be seen that the bilateral matching decision scheme of OI and TI of $\mathrm{M}$ enterprise is, $\mu_{M}{ }^{*}=\mu_{M \mathrm{t}}{ }^{*} \cup \mu_{M s}^{*} \quad$ where $\quad \mu_{M t}{ }^{*}=\left\{\left(A_{1}, B_{1}\right),\left(A_{1}, B_{2}\right),\left(A_{2}, B_{1}\right),\left(A_{3}, B_{1}\right),\left(A_{4}, B_{2}\right)\right\}$, $\mu_{M s}^{*}=\left\{\left(A_{2}, B_{2}\right),\left(A_{3}, B_{2}\right),\left(A_{4}, B_{1}\right)\right\}$. This result means that among all the OI and TI components of the $M$ enterprise, $A_{1}$ matches $B_{1}, A_{1}$ matches $B_{2}, A_{2}$ matches $B_{1}, A_{3}$ matches $B_{1}, A_{4}$ matches $B_{2}$, and $A_{2}$ does not match $B_{2}, A_{3}$ Does not match $B_{2}, A_{4}$ does not match $B_{1}$. 
The above empirical analysis results show that in the process of matching decision between OI and TI, the management decision makers of enterprise M should see that the strategic innovation of the enterprise is matched with the product innovation and process innovation. The organizational structure innovation is only matched with the product innovation. Cultural innovation matches product innovation, and organizational innovation only matches process innovation .The unmatched elements include organizational structure innovation and process innovation, organizational culture innovation and process innovation, organizational system innovation and product innovation. Therefore, the relevant management decision makers of enterprise $\mathrm{M}$ need to strengthen the optimization of the unmatched elements in the process of making optimal matching decisions between the OI and TI of the enterprise, and pay close attention to the matched element groups. Once a mismatch occurs, the firm needs to take targeted optimization measures immediately.

\section{Dynamic Matching Decisions between OI and TI of M Enterprise}

Based on the comprehensive survey data and data for M company in this research ${ }^{[7-8]}$, combined with relevant research contents in the life cycle of enterprises ${ }^{[9]}$, the development status of OI and TI of M company can be described, so that M companies can be judged It should be in the growth stage of the enterprise. The initial value of OI and TI of M enterprise can be set to 190 and 360 respectively, and the simulation period of the simulation is positioned for 10 years, that is, 120 months. On this basis, the system dynamics simulation method is used to bring the relevant values of M company into the VENSIM software, so as to obtain the simulation chart of the OI and TI matching process of $M$ company and the dynamic matching process of OI and TI ${ }^{[10]}$. The simulation diagram of the impact of M's innovation capability is shown in Figures 1 and 2. These two simulation diagrams can to a large extent express the current OI and TI development status of M company and the bilateral matching relationship.

As shown in Figure 1, overall, the simulation curves of TI and OI show a rising trend of varying degrees. Among them, the OI simulation curve is continuously rising under a certain degree of fluctuation, and the increase is relatively large. However, the TI simulation curve is always in a small rise phase, and the progress is very small. At the 98th month, the OI simulation curve and the TI simulation curve intersect. Before the 98th month, the overall level of TI's simulation curve was significantly higher than the level of OI's simulation curve. From the 99th month to the 120th month, the level of the OI simulation curve exceeded the level of the TI simulation curve. Judging from the matching development process and trend of OI and TI, the matching relationship between OI and TI has developed from a larger bilateral gap to a smaller bilateral gap. This also shows to a certain extent that the match between OI and TI of M company is getting better.

As shown in Figure 2, through the simulation and simulation curve of TI's impact on the enterprise's innovation capability, it can be seen that TI has a huge impact on the innovation capability of the enterprise, and has a positive effect, and the degree of the impact slowly increases. The impact of OI on corporate innovation capacity first increased, and the increase was relatively large, and it began to decline slowly in the 80th month. The degree of matching between OI and TI on the innovation capability of $\mathrm{M}$ enterprise is relatively close to that of $\mathrm{OI}$ on the innovation capabilities of enterprises, and the changes in their degree of influence are similar, but not exactly the same. It can be seen that in the current development stage of M enterprises, the impact of OI development of $\mathrm{M}$ enterprises on their innovation capabilities is closely related to their $\mathrm{TI}$ capabilities and levels.

In summary, through the above $\mathrm{M}$ simulation curve of OI and TI dynamic matching, we can see the current state of dynamic matching between OI and TI of M enterprise. Decision makers at the management level of $\mathrm{M}$ enterprise face the existence of dynamic matching between OI and TI of enterprise. The main problem can be used as a reference for decision-making, so as to draw a dynamic matching decision path map of MI OI and TI, as shown in Figure 3. The matching decision path of the M company's OI and TI should be as follows: First, based on the actual situation of the M company's OI and TI and related survey data, measure the OI and TI status of the M company, and then use the matrix bilateral matching decision evaluation method to determine the M company. 
Feature groups that are currently matched and unmatched. Therefore, it can be judged that the matched element group can have a certain promotion effect on the enterprise's innovation ability and even the enterprise performance to different degrees. For the already mismatched element groups, it is necessary to further optimize the matching status of organizational structure innovation and process innovation, the matching status of organizational cultural innovation and process innovation, and the matching relationship between organizational system innovation and product innovation. Based on the completion of the optimization, the OI and TI status of the M enterprise is measured again, and the matching status of the OI and TI of the M enterprise is determined.

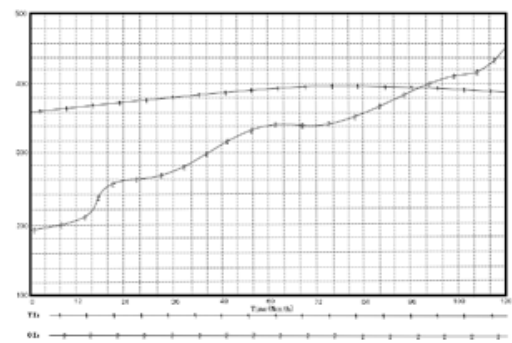

Fig.1 Simulation of matching process between OI and TI of M company

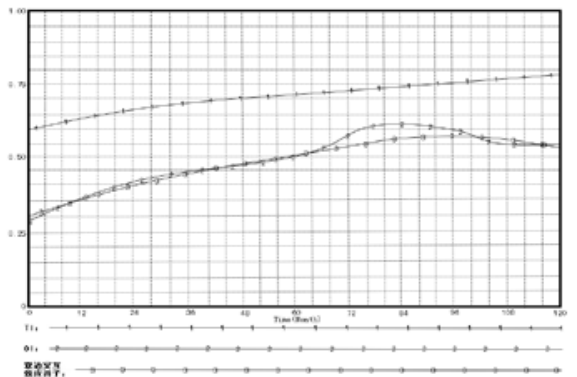

Fig.2 Simulation of matching process dynamic impact between OI and TI on innovation ability of M company

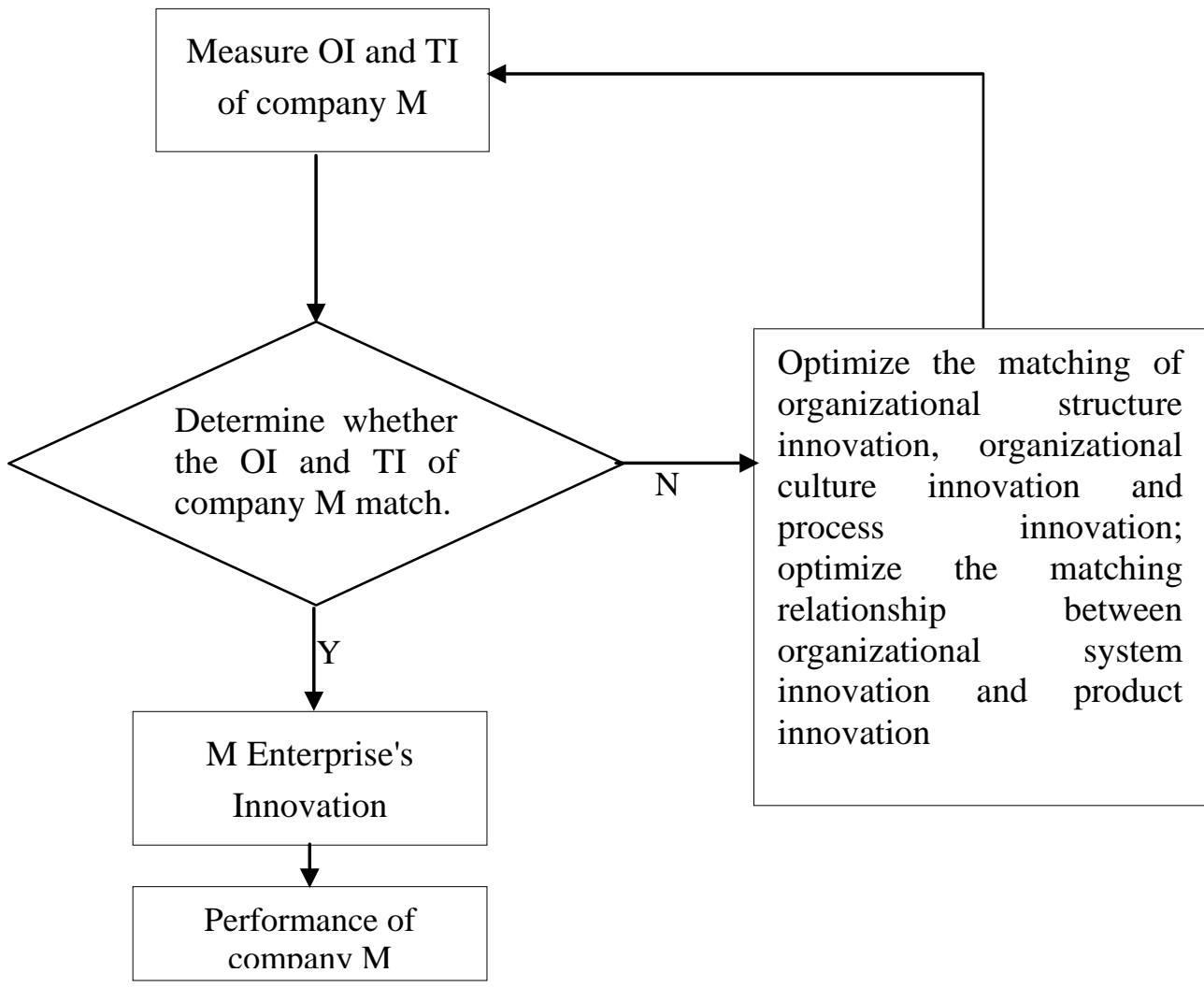

Fig.3 Decision route of matching relationship between OI and TI in M company 


\section{Suggestions for M Firm's OI and TI Matching Decisions}

Based on the previous analysis of the status of the OI and TI of the M enterprise and the bilateral matching status of the two, in order to further strengthen the effectiveness of the matching decision between the OI and the TI of the M enterprise, the author has made innovations, managers, and At the organizational level, the countermeasures and suggestions for matching implementation of MI OI and TI are put forward. The proposal of these countermeasures can also ensure the smooth development of the matching decision between OI and TI of M enterprises.

\subsection{Recommendations for implementation of decisions at the innovation level}

In the process of matching the decision between OI and TI, the ultimate purpose of the managers of enterprise $\mathrm{M}$ is to promote the improvement and progress of the overall comprehensive innovation capability of the enterprise, so as to comprehensively promote the realization of corporate performance through the improvement of the innovation capability of the enterprise. At the same time, the $\mathrm{M}$ enterprise's bilateral matching decision-making activities of OI and TI also need a certain innovation environment to support. Therefore, the author fully combines the actual development of OI and TI and their bilateral matching decisions, and proposes countermeasures and recommendations for the implementation of OI and TI matching decisions from the perspective of enterprise innovation.

Enterprise managers correctly take the following measures: clarifying the innovation goals of OI and TI of company M, and formulate a feasible corporate innovation strategy. Actively optimizing the innovation components of the MI OI and TI and the factors that influence bilateral innovation ${ }^{[11]}$. Improving the efficiency of dynamic management and control of innovation in the matching process between OI and TI.

\subsection{Recommendations for implementation of decisions at the managerial level}

In enterprise management practice, the management subject is still the manager of the enterprise ${ }^{[12]}$. Then the managers' personal qualities, decision-making methods, and managers' support for innovative activities will affect the managerial ability of enterprise managers to a large extent ${ }^{[13-14]}$. The same is true in $\mathrm{M}$ company. The basic situation of $\mathrm{M}$ company managers has a relatively important impact on the decision-making activities of OI and TI matching, and it will even have a decisive impact to a certain extent. Therefore, this research is based on the current status of innovation management practice of $\mathrm{M}$ enterprise, and puts forward countermeasures and recommendations for the implementation of matching decisions between OI and TI based on the comprehensive management level of $\mathrm{M}$ enterprise, in order to improve the management ability of enterprise managers to a certain extent.

Enterprise managers correctly take the following measures: Comprehensively improving the basic management quality of $\mathrm{M}$ enterprise managers. Further strengthening the scientific of the $\mathrm{M}$ enterprise management decision-making method. Strengthening the support of $M$ enterprise managers' support for enterprise innovation activities fundamentally speaking, the so-called innovation also means change, and change will inevitably be accompanied by risks to varying degrees ${ }^{[15]}$.

\subsection{Recommendations for implementation of decisions at the organizational level}

In the process of completing the decision-making management practice of OI and TI matching, $\mathrm{M}$ enterprise still needs to strengthen the management content at the organizational level. In particular, in the previous article, it was analyzed from a static perspective that in the practice of matching decision management between OI and TI, there are indeed some problems in the organizational level of OI. Therefore, the author proposes countermeasures for the implementation of matching decisions between OI and TI of $\mathrm{M}$ enterprise from an organizational level, in order to promote the smooth development of matching decision activities between OI and TI of M enterprise to a certain extent, consolidate the foundation of corporate management decisions, and strengthen corporate management decisions efficacy. 
Enterprise managers correctly take the following measures: further improving the organizational structure, organizational system and culture of $\mathrm{M}$ enterprise. Optimizing the organization's overall resource allocation and coordination ${ }^{[16]}$.

\section{Conclusion}

Through this study, it is found that there is indeed a certain obstacle in the implementation of the decision-making and management practice of the matching between organizational innovation and technological innovation in the case of enterprise $M$. In particular, the number of unmatched element groups is relatively large, thereby inhibiting enterprise innovation to a large extent. Sustainable development in practice. Therefore, the relevant managers of the $\mathrm{M}$ enterprise should actively take a number of measures to promote more scientific and effective management of the decision-making practice of corporate organizational innovation and technological innovation.

Of course, there are some deficiencies in the research of this paper. This study only selects $\mathrm{M}$ company as the research object for the matching decision of enterprise organizational innovation and technological innovation, which lacks certain representativeness. Therefore, future research can choose an industry as the research object, which has more extensive and application value.

\section{Acknowledgements}

This work was supported by the Basic scientific research business expense project of Heilongjiang provincial universities under Grant [number HDYJW201907].

\section{References}

[1] Xin C, Shi C S, Zheng-Gang W U. Research on the Tractive Impacts Among Structure oriented Organizational Innovation, Technological Innovation and Organizational Performance [J]. R \& D Management, 2008, 20(1):45-51.

[2] Wang Chenggang, Shi Chunsheng, Sun Honglie. Influencing Factors of Organizational Innovation of Chinese High-end Equipment Manufacturing Enterprises [J]. Science and Technology Management Research, 2017, 37 (15): 164-169. (Chinese)

[3] Camisón C, Villar-López A. Organizational innovation as an enabler of technological innovation capabilities and firm performance [J]. Journal of Business Research, 2014, 67(1):2891-2902.

[4] Zhang Meili, Shi Chunsheng. An Empirical Study on the Division of Organizational Innovation Dimensions in High-tech Manufacturing Enterprises [J]. China Science and Technology Forum, 2015 (4): 68-74.(Chinese)

[5] Miriam A. Drake. Technological Innovation and Organizational Change [J]. Journal of Academic Librarianship, 2000, 26(1):53-59.

[6] Roberts E B. Stimulating Technological Innovation: Organizational Approaches [J]. Research management, 1979, 22(6):26-30.

[7] Miriam A. Drake. Technological Innovation and Organizational Change [M]. Technological change and organization /. Edward Elgar Pub. 1994:53-59.

[8] Aloini D, Lazzarotti V, Manzini R, et al. Implementing Open Innovation: Technological, Organizational and Managerial tools [J]. Business Process Management Journal, 2017, 23(6):10-20.

[9] Zhang Meili, Shi Chunsheng, Jia Yunqing, et al. Research on the matching mechanism between OI and TI of enterprises in different life cycle stages [J]. Studies in Science of Science, 2015, 33 (8): 1261-1270.(Chinese)

[10] Neriman - Esendemir, Zehir C. Innovation and Performance: The Mediating Effect of 
Organizational Learning and Technological Capability [J]. 2017, 6(4):48.

[11] Azar G, Ciabuschi F. Organizational innovation, technological innovation, and export performance: The effects of innovation radicalness and extensiveness [J]. International Business Review, 2016, 26(2):324-336.

[12] Li Jing, Shi Chunsheng, Liu Weiwei. Measurement Research on the Matching State of Organizational Innovation and Technology Innovation in High-tech Enterprises [J]. Journal of Management Engineering, 2011, 25 (4): 172-177. (Chinese)

[13] Staropoli C. Cooperation in R\&D in the pharmaceutical industry - The network as an organizational innovation governing technological innovation [J]. Technovation, 1998, 18(1):13-23.

[14] Zhang Dapeng, Sun Xinbo, Qian Yu. The influence of the matching of leadership style and organizational innovation strategy on the transformation and upgrading of enterprises [J]. Technology Economy, 2017, 36 (3): 79-88. (Chinese)

[15] Liang Shuanglu, Zhang Mei. Research on the Coordination of Industrial Innovation, Technical Innovation and Organizational Innovation in Eastern, Central and Western Regions of China [J]. Science \& Technology Progress and Policy, 2017, 34 (10): 33-38. (Chinese)

[16] Zhan Weiping, Shen Qunhong, Yu Yongda. Analysis on the Synergy between Technological Innovation and Organizational Reform: Based on the Perspective of Organizational Knowledge Distribution [J]. China Science and Technology Forum, 2010 (6): 31-36. (Chinese) 